\title{
Correlation of monocyte/HDL ratio (MHR) with inflammatory parameters in obese patients diagnosed with polycystic ovary syndrome
}

\author{
Dilsad Herkiloglu(D, Sefik Gokce \\ Istanbul Yeni Yuzyil University Department Of Obstetrics And Gynecology, Istanbul, Turkey
}

\begin{abstract}
Objectives: Monocyte/high density lipprotein (HDL) ratio (MHR) has been reported to be associated with obesity and polycystic ovarian syndrome (PCOS). In this study, it was aimed to evaluate whether there is a relationship between PCOS and MHR and inflammatory parameters, to investigate the relationship level of MHR and lymphocyte/monocyte ratio (LMR), which are easily accessible inflammatory and oxidative stress markers, with obese women with PCOS, and to determine the usability of MHR as a predictive marker for PCOS.

Material and methods: The study included 64 PCOS-patients who were admitted to Gynecology clinics and 52 healthy women.

Results: The mean MHR $(12.5 \pm 4.6)$ in the PCOS group was significantly higher than the control group $(10.4 \pm 4.0)(p=0.01)$. In the examination performed by combining the groups PCOS and obesity status, the mean MHR value in the PCOS-obese group was significantly higher than all the other groups $(p=0.004)$. In the ROC analysis, the threshold value of 10.1 for MHR was found to have a sensitivity of $84.8 \%$ and specificity of $58.5 \%$ in determining the association between PCOS and obesity (AUC: $0.721 ; p<0.001 ;$ LB: 0.628 ; UB: $0.814 ;$ Cl 95\%). Accordingly, the rate of those with MHR level of 10.1 and above was significantly higher in the PCOS group compared to the control group ( $67.2 \%$ vs $40.4 \%)(p=0.001)$. In the logistic regression analysis, the determination is increased by 3,026 times (odds ratio; 1.401-6.535) in predicting the presence of PCOS in those with MHR value of 10.1 and above, and 7,576 times (Odds ratio; 2.652-21.646) in predicting the presence of PCOS + obesity. was found to be. Correlation analysis in PCOS patients revealed that the MHR value was negatively correlated with age $(p=0.001 ; r=-0.412), \operatorname{LMR}(p=0.003 ; r=-0.377)$, and total cholesterol $[p=0.018$; correlation coefficient $(r)=-0.302]$.

Conclusions: This study findings showed that MHR level is significantly related to PCOS, and especially MHR values above 10.1 may be a significant predictive marker for PCOS. Our study findings also show that an association of PCOS and obesity is a very important trigger on MHR.
\end{abstract}

Key words: monocyte/HDL ratio; PCOS; obesity

Ginekologia Polska 2021; 92, 8: 537-543

\section{INTRODUCTION}

Polycystic ovary syndrome (PCOS) is a disorder characterized by impaired hormone balance, polycystic ovaries and ovulation disorders disorder, such as hyperandrogenism. Impaired hormone balance in PCOS affects many systems, causing a wide variety of complications. It has been reported that the frequency of obesity increases in women with PCOS, and that PCOS can cause dyslipidemia and cardiovascular diseases more frequently in these patients $[1,2]$.

Monocytes are one of the main structures in the immune system. It has been suggested that monocytes play a role in atherosclerosis. High-density lipoproteins (HDL) are known to play a role in the prevention of atherosclerosis and cardiovascular diseases. It has been reported that HDL also affects the functions of monocytes and functions to prevent monocytes from functioning in atherosclerosis and cardiovascular disease [3-5]. In this context, the monocyte/ /HDL ratio (MHR) has been shown to increase in many diseases, especially in atherosclerosis and cardiovascular diseases, and it has been shown that MHR can be used as a predictive marker in many disorders $[5,6]$.

It has been reported that MHR is associated with metabolic syndrome, obesity and PCOS [7, 8]. However, there are very few studies showing a direct relationship between

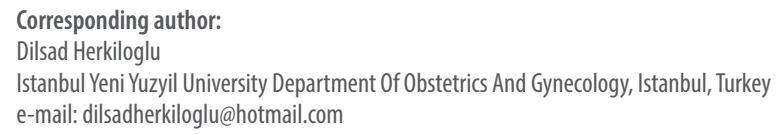


MHR with PCOS and obesity. It has been proven that obesity, which has an important place in cardiovascular diseases, is significantly and independently associated with high MHR and low LMR levels [9]. In this study, we aimed to evaluate whether there is a relationship between PCOS and MHR and other inflammatory parameters, to investigate the relationship of MHR and LMR that are easily obtained inflammatory and oxidative stress markers, with obesity in women with PCOS, and to determine the usability of MHR as a predictive marker for PCOS.

\section{MATERIAL AND METHODS}

Patients and Tests

The study included 64 patients diagnosed with PCOS and 52 healthy women who presented to the Gynecology and Obstetrics clinics of our tertiary hospital. The study was approved by the ethics committee in accordance with the requirements of the Declaration of Helsinki. Informed consent was obtained from all participants. PCOS was diagnosed using Rotterdam criteria [10]. According to these criteria; oligomenorrhea (where menstrual cycles occur for more than 35 days) or amenorrhea (where menstrual cycles occur within at least six months intervals) is defined as oligoovulation. In addition, the presence of hirsutism, one of the hyperandrogenism findings, was evaluated using the Ferriman-Gallwey scoring system [11]. Physical and gynecological examinations, pelvic ultrasounds and peripheral venous blood sampling were performed on the second or third day of a participant's menstrual cycles. All women were examined and pelvic ultrasound scans were performed by the same gynecologist using a $7.0 \mathrm{MHz}$ vaginal transducer (Voluson 730, GE Healthcare, USA).

Patients who received medications for some diseases such as Cushing syndrome, congenital adrenal hyperplasia, androgen-secreting tumors, oral contraceptives, antilipidemic and/or antihypertensive drugs, steroids, diabetic drugs, anticoagulants or antiplatelet drugs were excluded from the study.

After one night fasting, blood samples of the patients were taken from the antecubital veins. For serum, biochemical and hormonal evaluation; complete blood counts were measured using fluorescent flow cytometry or electrical impedance method. Serum levels of follicle-stimulating hormone (FSH), luteinizing hormone (LH), oestradiol (E2), thyroid-stimulating hormone (TSH), antimullient hormone and total testosterone were determined using commercially available enzyme-dependent immunosorbent assay (ELISA) kits (eBioscience). Glucose, insulin, total cholesterol, low density lipoprotein (LDL) cholesterol and high density lipoprotein (HDL) cholesterol levels were measured with AutoAnalyzer. Those with a BMl value $\geq 25 \mathrm{~kg} / \mathrm{m}^{2}$ were considered obese [12]. The patients were divided into four groups according to the presence or absence of PCOS and obesity [8]. Accordingly, 38 obese patients diagnosed with PCOS and 35 obese patients without a diagnosis of PCOS were included in the study.

The LMR value was calculated by dividing the absolute lymphocyte by the absolute number of monocytes. The basic MHR was calculated by dividing the number of monocytes by the HDL-C level.

Depending on the result of the receiver operating characteristic (ROC) analysis performed on the MHR value, the participants were divided into two groups according to a 10.1 cut-off value.

\section{Statistical analysis}

All statistical analyzes were performed using SPSS version 25.0 software (IBM SPSS, Chicago, IL, USA). Descriptive data are given as numbers and percentages. Comparisons between the groups were made with Pearson's Chi Square test and Fisher's Exact Test for the categorical variables. Normality of the continuous variables was evaluated with the Kolmogorov-Smirnov Test. The differences between the groups in terms of continuous variables were analyzed using Student's t Test, and the comparison of mean values between multiple groups by variance analysis. The relationships between continuous variables were tested using Spearman's correlation analysis. The capacity of MHR to predict the association of PCOS and obesity was analyzed using ROC curve analysis. Risk coefficient of categorical variables was evaluated by logistic regression analysis and given as "odds ratio". The results were evaluated within the $95 \%$ confidence interval, and $p<0.05$ values were considered statistically significant. Bonferroni correction was made where appropriate.

\section{RESULTS}

The mean age was $26.5 \pm 3.8$ years (range: $20-34$ years) in the PCOS group, $27.8 \pm 3.6$ (range: $21-34$ years) in the control group, and there was no significant difference between both groups in terms of mean age $(p=0.077)$. The mean MHR (12.5 \pm 4.6$)$ in the PCOS group was statistically significantly higher than the control group (10.4 \pm 4.0$)$ $(p=0.01)$ (Tab. 1).

In the examination performed by combining PCOS and obesity status of the groups, the mean MHR value was significantly higher in the PCOS-obese group compared to all other groups ( $p=0.004$ ) (Tab. 2, Fig. 1).

In the ROC analysis, cut-off value of 10.1 for MHR was found to have a sensitivity of $84.8 \%$ and a specificity of $58.5 \%$ in determining the association between PCOS and obesity [area under curve (AUC): 0.721; $\mathrm{p}<0.001$; lower bound (LB): 0.628; upper bound (UB): 0.814; confidence interval (CI) 95\%] (Fig. 2). Accordingly, the rate of those with a MHR level $\geq 10.1$ was significantly higher in the PCOS group compared to the control group 


\begin{tabular}{|c|c|c|c|c|c|}
\hline & \multicolumn{2}{|c|}{$\begin{array}{l}\text { PCOS } \\
(n=64)\end{array}$} & \multicolumn{2}{|c|}{$\begin{array}{l}\text { Control } \\
(n=52)\end{array}$} & \multirow{2}{*}{$\mathbf{p}$} \\
\hline & Mean & SD & Mean & SD & \\
\hline Total cholesterol [mg/dL] & 183.1 & 32.4 & 171.7 & 26.0 & 0.042 \\
\hline Monocyte/HDL ratio & 12.5 & 4.6 & 10.4 & 4.0 & 0.010 \\
\hline $\begin{array}{l}\text { Neutrophil/lymphocyte } \\
\text { ratio }\end{array}$ & 1.9 & 0.6 & 2.2 & 1.2 & 0.040 \\
\hline $\begin{array}{l}\text { Lymphocyte/monocyte } \\
\text { ratio }\end{array}$ & 4.5 & 1.7 & 4.5 & 1.7 & 0.926 \\
\hline Age [years] & 26.5 & 3.8 & 27.8 & 3.6 & 0.077 \\
\hline Glucose [mg/dL] & 91.4 & 12.8 & 91.8 & 8.3 & 0.856 \\
\hline Insulin $[\mu \mid \mathrm{U} / \mathrm{mL}]$ & 11.9 & 7.2 & 9.3 & 5.8 & 0.033 \\
\hline LDL cholesterol [mg/dL] & 118.6 & 29.5 & 108.6 & 27.0 & 0.066 \\
\hline $\begin{array}{l}\text { Anti-Müllerian Hormon } \\
{[\mathrm{mg} / \mathrm{dL}]}\end{array}$ & 11.7 & 8.1 & 5.1 & 14.1 & 0.002 \\
\hline Trigliserid [mg/dL] & 113.7 & 68.9 & 85.5 & 50.0 & 0.017 \\
\hline WBC $\left[10^{9} / \mathrm{L}\right]$ & 7.5 & 1.7 & 7.2 & 2.1 & 0.352 \\
\hline Neutrophils $\left[10^{9} / \mathrm{L}\right]$ & 4.2 & 1.2 & 4.2 & 1.2 & 0.960 \\
\hline Lymphocytes $\left[10^{9} / L\right]$ & 2.4 & 0.6 & 2.1 & 0.9 & 0.113 \\
\hline $\operatorname{RBC}\left[10^{9} / \mathrm{L}\right]$ & 6.0 & 1.8 & 4.5 & 0.3 & $<0.001$ \\
\hline MPV [fL] & 7.7 & 0.9 & 8.0 & 1.0 & 0.085 \\
\hline $\mathrm{HBa} 1 \mathrm{c}[\mathrm{mmol} / \mathrm{mol}]$ & 5.4 & 0.4 & 5.5 & 0.3 & 0.432 \\
\hline
\end{tabular}

PCOS - polycystic ovary syndrome; MHR - monocyte/high density lipoprotein ratio; $L D L$ - low-density lioprotein; $R B C$ - red blood cells; WBC - white blood cells; MPV - mean platelet volume; SD - standard deviation

(67.2\% vs $40.4 \%)(p=0.001)$. In addition, the rate of those with a MHR level $\geq 10.1$ in the PCOS-obese group was significantly higher than the other groups (by combining groups) (84.8\% vs $42.5 \%$ ) ( $p<0.001)$. In the logistic regression analysis, the determination of PCOS was increased by 3.026 folds (odds ratio; 1.401-6.535) in predicting the presence of PCOS compared to those below 10.1, and 7.576 folds (odds ratio; 2.652-21.646) in predicting the presence of PCOS + obesity (Tab. 3).

Correlation analysis in PCOS patients revealed that MHR value was negatively correlated with age $(p=0.001$; $r=-0.412), \operatorname{LMR}(p=0.003 ; r=-0.377)$ and total cholesterol $(p=0.018 ; r=-0.302)($ Tab. 4$)$.

\section{DISCUSSION}

Polycystic ovarian syndrome is a syndrome that can affect all body systems including the cardiovascular system $[1,2,13]$. Obesity, which is more common in patients with PCOS, also increases the cardiovascular risk [12-14]. While several studies in the literature $[5,6]$ have shown that MHR, which has been recently investigated, is an indicator of atherosclerosis and cardiovascular disease, the present study demonstrated that MHR is significantly associated with both PCOS and the association between PCOS and obesity.
Inflammation and lipid accumulation are two indicators of atherosclerosis. In addition to being localized, inflammation is a systemic process with increased levels of mediator molecules. Monocytes are a resource for structures involved in the proinflammatory process. Monocytes play a role in the first stage of progression of atherosclerosis. They take part in the immune-mediated process, and bind to adhesion molecules in the damaged vascular endothelium, migrate to the subendothelial cavity, where they mature and differentiate into macrophages [5, 15]. Modified LDLs in atherosclerosis are taken up by macrophages located in the vascular wall, inducing the release of inflammatory cytokines. Monocytes also play a role in atherogenesis and therefore in the pathogenesis of cardiovascular diseases through many different mechanisms $[5,16]$. HDL neutralizes the proinflammatory effect of monocytes by inhibiting the migration of macrophages and LDL oxidation as well as the removal of cholesterol from the cells. HDL reduces the content of F-actin and prevents monocytes from functioning in the arterial wall. It also prevents the adhesion of monocytes to the endothelial wall $[5,17]$. HDL also suppresses activation of monocytes and transformation of precursor cells into monocytes [5]. It has been suggested that the inverse of this mechanism, monocyte accumulation and decrease in HDL may play a role in atherosclerosis and cardiovascular diseases. In this context, it has been stated that HDL plays a role in the reduction of atherosclerosis $[5,18,19]$.

It was suggested that an increase in HDL and a decrease of monocytes had a positive effect on atherosclerosis, and the MHR value calculated by the ratio of monocyte count to HDL level could be a negative predictive marker for atherosclerosis and cardiovascular diseases $[5,16]$. MHR has been shown to be a prognostic factor for cardiovascular diseases [5, 16, 18]. MHR has also been reported to show asymptomatic organ damage, systemic inflammation and nephropathy in patients with hypertension [19, 20]. Usta et al. [8] and Vahit et al. [9], showed that MHR was a marker of metabolic syndrome characterized by obesity, increased glucose, blood pressure, LDL and decreased HDL. In a meta-analysis and systematic review of 23 studies, Wekker et al. found that women with PCOS are more likely to be diagnosed with cardiometabolic risk factors such as Type 2 diabetes mellitus and hypertension compared to women without PCOS. The authors also concluded that women with PCOS are at a higher risk of developing non-fatal cerebrovascular events. Sensitivity meta-analyses of high-quality studies have proven that the risk of developing Type 2 DM and $\mathrm{HT}$ are higher in women with PCOS compared to those without PCOS [21]. In another meta-analysis, a slight correlation was shown between biochemical parameters of hyperandrogenism and metabolic findings, while some correlation was found with these parameters and women 
Table 2. Comparison of average values between groups

\begin{tabular}{|c|c|c|c|c|c|c|c|c|c|c|c|}
\hline & \multicolumn{2}{|c|}{ PCOS-Obese } & \multicolumn{2}{|c|}{ PCOS-Lean } & \multicolumn{2}{|c|}{ Control-Obese } & \multicolumn{2}{|c|}{ Control-Lean } & \multicolumn{2}{|l|}{ Total } & \multirow{2}{*}{$\mathbf{p}$} \\
\hline & Mean & SD & Mean & SD & Mean & SD & Mean & SD & Mean & SD & \\
\hline Age [years] & 26.7 & 3.7 & 26.3 & 3.9 & 28.8 & 4.0 & 27.4 & 3.5 & 27.1 & 3.8 & 0.184 \\
\hline Monocyte/HDL ratio & 13.9 & 4.8 & 11.4 & 3.9 & 10.3 & 4.1 & 10.4 & 4.0 & 11.5 & 4.4 & 0.004 \\
\hline $\begin{array}{l}\text { Neutrophil/lymphocyte } \\
\text { ratio }\end{array}$ & 1.9 & 0.6 & 1.9 & 0.7 & 2.2 & 1.0 & 2.2 & 1.3 & 2.0 & 0.9 & 0.240 \\
\hline $\begin{array}{l}\text { Lyphocyte/monocyte } \\
\text { ratio }\end{array}$ & 4.6 & 1.8 & 4.4 & 1.6 & 5.1 & 1.7 & 4.2 & 1.7 & 4.5 & 1.7 & 0.367 \\
\hline Total cholesterol [mg/dL] & 186.3 & 31.7 & 179.0 & 33.5 & 175.5 & 20.4 & 170.2 & 28.1 & 178.0 & 30.2 & 0.146 \\
\hline Glucose [mg/dL] & 92.6 & 15.5 & 89.8 & 8.0 & 94.6 & 5.4 & 90.6 & 9.1 & 91.5 & 11.0 & 0.480 \\
\hline Insulin $[\mu \mathrm{IU} / \mathrm{mL}]$ & 13.6 & 6.4 & 9.8 & 7.7 & 10.3 & 5.7 & 8.9 & 5.9 & 10.7 & 6.7 & 0.016 \\
\hline LDL Cholesterol [mg/dL] & 115.3 & 27.6 & 122.6 & 31.6 & 113.6 & 23.6 & 106.5 & 28.4 & 114.1 & 28.7 & 0.169 \\
\hline $\begin{array}{l}\text { Anti-Müllerian Hormon } \\
{[\mathrm{mg} / \mathrm{dL}]}\end{array}$ & 11.2 & 7.7 & 12.2 & 8.8 & 9.2 & 26.1 & 3.4 & 3.0 & 8.7 & 11.7 & 0.007 \\
\hline Triglyceride [mg/dL] & 125.6 & 77.7 & 99.2 & 54.2 & 89.5 & 33.3 & 83.8 & 56.0 & 101.1 & 62.5 & 0.035 \\
\hline Neutrophils [10/L] & 4.3 & 1.1 & 4.1 & 1.5 & 4.7 & 1.2 & 4.1 & 1.2 & 4.2 & 1.2 & 0.431 \\
\hline Lymphocytes [109/L] & 2.4 & 0.6 & 2.3 & 0.6 & 2.3 & 0.7 & 2.1 & 0.9 & 2.3 & 0.7 & 0.238 \\
\hline Monocytes [109/L] & 0.6 & 0.2 & 0.5 & 0.2 & 0.5 & 0.2 & 0.5 & 0.2 & 0.5 & 0.2 & 0.200 \\
\hline $\operatorname{RBC}\left[10^{9} / \mathrm{L}\right]$ & 6.1 & 1.9 & 6.0 & 1.7 & 4.6 & 0.4 & 4.5 & 0.3 & 5.3 & 1.5 & $<0.001$ \\
\hline WBC $\left[10^{9} / L\right]$ & 7.6 & 1.4 & 7.3 & 2.1 & 7.8 & 2.1 & 6.9 & 2.0 & 7.3 & 1.9 & 0.263 \\
\hline MPV [fL] & 7.9 & 0.8 & 7.5 & 1.0 & 8.1 & 1.3 & 8.0 & 1.0 & 7.9 & 1.0 & 0.134 \\
\hline $\mathrm{HBa} 1 \mathrm{c}[\mathrm{mmol} / \mathrm{mol}]$ & 5.4 & 0.3 & 5.4 & 0.5 & 5.5 & 0.3 & 5.5 & 0.3 & 5.4 & 0.4 & 0.869 \\
\hline
\end{tabular}

${ }^{*} \mathrm{p}<0.008$ values were considered significant according to the Bonferroni correction; PCOS - polycystic ovary syndrome; MHR - monocyte/high density lipoprotein ratio; LDL — low-density lioprotein; RBC — red blood cells; WBC — white blood cells; MPV — mean platelet volume

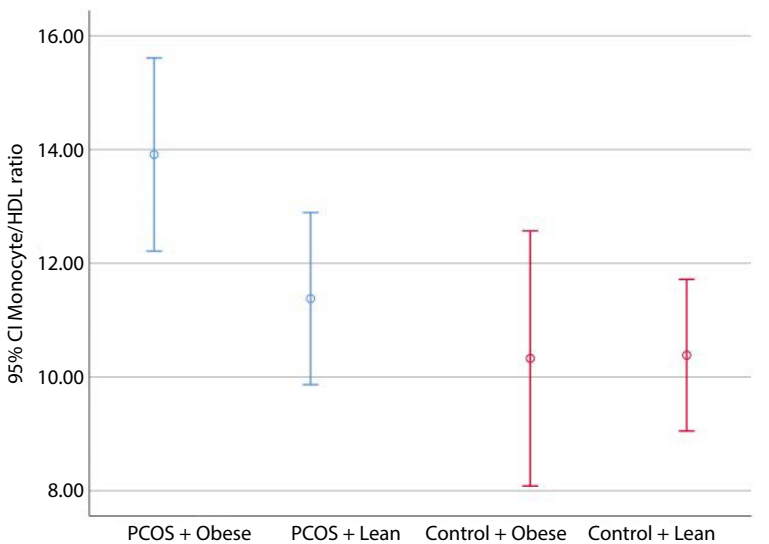

Figure 1. Clustering of $\mathrm{MHL}$ - monocytye/high-density lipoprotein values by groups; $\mathrm{Cl}$ - confidence interval; $\mathrm{HDL}$ - high density lipprotein; PCOS - polycystic ovary syndrome

with PCOS. A correlation was shown only with $\mathrm{fT}$, insulin levels and insulin resistance, but it was reported that this correlation might be due to the well-known effect of insulin on SHBG and it was attributed to the possible limited effect of excess insulin on androgen ovarian production [22].

Increased inflammatory neutrophils cause the release of several types of cytokines, proteolytic enzymes, leading to tissue damage through activation of the coagula-

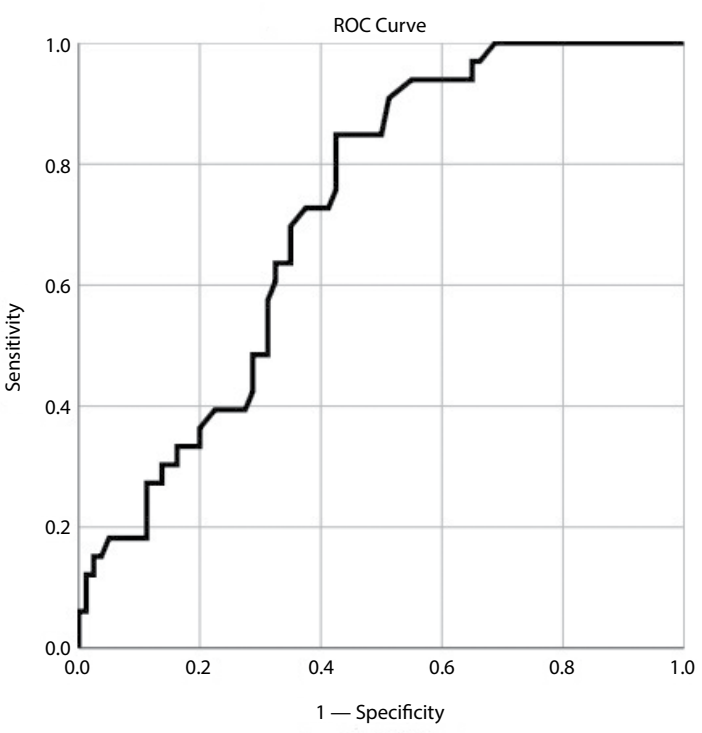

Figure 2. In the receiver operating characteristic analysis, the threshold value of 10.1 for monocyte/high density lipprotein ratio was found to have a sensitivity of $84.8 \%$ and specificity of $58.5 \%$ in determining the association between polycystic ovary syndrome and obesity (AUC: 0.721 ; p < 0.001; LB: 0.628; UB: 0.814; CI 95\%); ROC — receiver operating characteristic

tion cascade and impairment of endothelial integrity [23]. In response to inflammatory conditions, the number of 
Table 3. Comparison between groups by taking monocyte/high density lipoprotein ratio threshold value (determined by ROC analysis) 10.1

\begin{tabular}{|c|c|c|c|c|c|c|}
\hline \multirow{3}{*}{ MHR* $^{*}$} & \multicolumn{4}{|c|}{ Groups } & p & OR \\
\hline & \multicolumn{2}{|c|}{ PCOS } & \multicolumn{2}{|c|}{ Control } & \multirow[t]{2}{*}{0.004} & \multirow{2}{*}{$\begin{array}{l}3.026 \\
(1.401-6.535)\end{array}$} \\
\hline & $\mathrm{n}$ & $\%$ & $n$ & $\%$ & & \\
\hline$<10.1$ & 20 & 32.8 & 31 & 59.6 & & \\
\hline \multirow[t]{2}{*}{$\geq 10.1$} & 41 & 67.2 & 21 & 40.4 & & \\
\hline & \multicolumn{2}{|c|}{ PCOS-Obese ${ }^{* *}$} & \multicolumn{2}{|c|}{ Other groups } & $<0.001$ & $\begin{array}{l}7.576 \\
(2.652-21.646)\end{array}$ \\
\hline$<10.1$ & 5 & 15.2 & 46 & 57.5 & & \\
\hline$\geq 10.1$ & 28 & 84.8 & 34 & 42.5 & & \\
\hline
\end{tabular}

*MHR groups are formed according to the threshold that the ROC analyze revealed the ghighest sensitivity and specificity; MHR - monocyte/high density lipoprotein ratio; PCOS - polycystic ovary syndrome; OR — odds lymphocytes decreases, and it has been associated with adverse prognosis in coronary artery disease [24]. Activation of monocytes is associated with inflammation and atherosclerosis [25]. Monocytes and structurally altered macrophages trigger the production of other inflammatory cytokines $[25,26]$. Systemic inflammation can be measured using various biochemical and hematological markers [27]. LMR has been proposed as a substitute marker for inflammation in different populations, and also has a prognostic and predictive value [28, 29]. Yue et al. [30], investigated the neutrophil-lymphocyte ratio and the predictive value of the monocyte-lymphocyte ratio in diabetic retinopathy. In that study, no relationship was found between the severity of diabetic retinopathy and the monocyte/lymphocyte ratio, but the monocyte/lymphocyte ratio was found to be an independent risk factor for diabetic retinopathy [30].

Table 4. Correlation analysis between laboratory findings within the polycystic ovary syndrome group

\begin{tabular}{|c|c|c|c|c|c|c|c|c|}
\hline & & Age & $\begin{array}{l}\text { Monocyte/ } \\
\text { HDL ratio }\end{array}$ & $\begin{array}{l}\text { Neutrophil/ } \\
\text { lymphocyte ratio }\end{array}$ & $\begin{array}{l}\text { Lyphocyte / } \\
\text { monocyte ratio }\end{array}$ & $\begin{array}{l}\text { Total } \\
\text { cholesterol }\end{array}$ & $\begin{array}{l}\text { Anti-Müllerian } \\
\text { Hormon }\end{array}$ & Triglyceride \\
\hline \multirow{2}{*}{ Monocyte/HDL ratio } & r & $-0.412^{* *}$ & & & & & & \\
\hline & $p$ & 0.001 & & & & & & \\
\hline \multirow{2}{*}{$\begin{array}{l}\text { Neutrophi//lymphocyte } \\
\text { ratio }\end{array}$} & $r$ & -0.207 & 0.088 & & & & & \\
\hline & $p$ & 0.101 & 0.500 & & & & & \\
\hline \multirow{2}{*}{$\begin{array}{l}\text { Lymphocyte/monocyte } \\
\text { ratio }\end{array}$} & $r$ & 0.182 & $-0.377^{* *}$ & $-0.355^{* *}$ & & & & \\
\hline & $p$ & 0.149 & 0.003 & 0.004 & & & & \\
\hline \multirow{2}{*}{ Total cholesterol } & r & 0.072 & $-0.302^{*}$ & 0.074 & 0.172 & & & \\
\hline & $p$ & 0.570 & 0.018 & 0.559 & 0.175 & & & \\
\hline \multirow{2}{*}{ Anti-Müllerian Hormon } & r & -0.070 & -0.058 & $-0.301^{*}$ & 0.155 & 0.017 & & \\
\hline & $p$ & 0.587 & 0.659 & 0.016 & 0.226 & 0.893 & & \\
\hline \multirow{2}{*}{ Triglyceride } & r & -0.190 & $0.303^{*}$ & 0.063 & 0.205 & $0.303^{*}$ & 0.019 & \\
\hline & $p$ & 0.140 & 0.020 & 0.627 & 0.110 & 0.017 & 0.885 & \\
\hline \multirow{2}{*}{ BMI } & r & 0.221 & 0.161 & -0.017 & 0.139 & 0.016 & -0.020 & 0.214 \\
\hline & $p$ & 0.079 & 0.214 & 0.896 & 0.275 & 0.900 & 0.878 & 0.095 \\
\hline \multirow{2}{*}{ WBC } & r & -0.126 & 0.190 & $0.360^{* *}$ & $0.337^{* *}$ & 0.202 & -0.099 & $0.310^{*}$ \\
\hline & $\mathrm{p}$ & 0.319 & 0.142 & 0.004 & 0.006 & 0.110 & 0.442 & 0.014 \\
\hline \multirow{2}{*}{ Neutrophils } & r & -0.218 & 0.195 & $0.648^{* *}$ & 0.138 & 0.116 & -0.238 & $0.280^{*}$ \\
\hline & $p$ & 0.083 & 0.131 & $<0.001$ & 0.277 & 0.363 & 0.060 & 0.027 \\
\hline \multirow{2}{*}{ Lymphocytes } & $r$ & -0.033 & 0.183 & $-0.460^{* *}$ & $0.671^{* *}$ & 0.084 & 0.116 & $0.270^{*}$ \\
\hline & $p$ & 0.794 & 0.159 & $<0.001$ & $<0.001$ & 0.508 & 0.366 & 0.034 \\
\hline \multirow{2}{*}{ Monocytes } & r & $-0.302^{*}$ & $0.784^{* *}$ & 0.084 & $-0.616^{* *}$ & -0.174 & -0.043 & -0.053 \\
\hline & $p$ & 0.015 & $<0.001$ & 0.508 & $<0.001$ & 0.169 & 0.738 & 0.683 \\
\hline \multirow{2}{*}{$\mathrm{RBC}$} & $r$ & -0.185 & -0.081 & 0.046 & 0.021 & $0.302^{*}$ & $0.435^{* *}$ & 0.132 \\
\hline & $p$ & 0.144 & 0.532 & 0.719 & 0.868 & 0.015 & $<0.001$ & 0.306 \\
\hline \multirow{2}{*}{ MPV } & $r$ & 0.230 & -0.223 & 0.051 & 0.137 & 0.166 & 0.028 & -0.030 \\
\hline & $p$ & 0.067 & 0.084 & 0.690 & 0.279 & 0.189 & 0.829 & 0.820 \\
\hline \multirow{2}{*}{ Homosistein } & $r$ & -0.105 & -0.001 & -0.137 & -0.019 & -0.228 & 0.084 & -0.129 \\
\hline & $p$ & 0.411 & 0.996 & 0.282 & 0.883 & 0.070 & 0.513 & 0.316 \\
\hline
\end{tabular}

$\mathrm{HDL}$ — high-density lioprotein; BMI — body mass index; WBC — white blood cells; RBC — red blood cells; MPV — mean platelet volume 
LMR has also been observed to be associated with vascular pathologies such as coronary artery disease and peripheral vascular disease [31]. As a result of our study, we found that MHR value was negatively correlated with $L M R(p=0.003$; $r=-0.377$ ) in PCOS patients who are at cardiovascular risk. According to this finding, as LMR decreases, MHR value increases significantly in patients with PCOS. In conclusion, our findings support that patients with PCOS can be followed up using LMR and MHR parameters for cardiovascular risk.

Dincgez-Cakmak et al. [7], found a significantly higher mean MHR value in PCOS patients compared to the control group (9.6 vs 8.2) and reported a significant relationship between MHR and PCOS for the first time. However, these researchers additionally found that the mean MHR value in PCOS patients with metabolic syndrome was significantly higher than PCOS patients without metabolic syndrome, and stated that MHR value could be used as a predictive marker for metabolic syndrome in PCOS. For this reason, in our study in which we aimed to evaluate the relationship between PCOS and MHR, patients with metabolic syndrome were excluded from the study. Usta et al. [13], found a significantly higher mean MHR value in patients with PCOS than the control group (11.5 and 8.8). In our study, the mean MHR in the PCOS group $(12.5 \pm 4.6)$ was significantly higher than the control group $(10.4 \pm 4.0)(p=0.01)$. In the ROC analysis conducted in our study, the sensitivity of a cut-off value of 10.1 for MHR was found as $84.8 \%$ and specificity as $58.5 \%$ in determining the association between PCOS and obesity (AUC: 0.721; $<<0.001$; LB: 0.628; UB: 0.814; $\mathrm{Cl} 95 \%$ ). Accordingly, the rate of those who had a MHR level $\geq 10.1$ in the PCOS group was found to be significantly higher than the control group (67.2\% vs $40.4 \%)(p=0.001)$. All these data show that MHR increases significantly in PCOS patients, especially MHR values above 10.1 may have a high sensitivity rate in detection of patients with PCOS.

Usta et al. [13], calculated in their logistic regression analysis that the MHR value increased by 1.101 folds increased determination power in predicting the presence of PCOS. In the logistic regression analysis performed in our study, it was calculated that there was a determination power of 3.026 folds (odds ratio; $1.401-6.535$ ) in predicting the presence of PCOS in those with a MHR value $\geq 10.1$. This finding shows that there is a clear relationship between MHR and PCOS.

Usta et al. [13], stated that MHR had an association not only with PCOS but also with obesity. These researchers divided the patients into four groups according to the presence of both PCOS and obesity, and found that the mean MHR was significantly higher in the PCOS-obese group than in all other groups. These researchers also found that the mean MHR in the obese control group was higher than the non-obese controls. In our study, according to the presence of both PCOS and obesity, the patients were divided into four groups and analyzed. Thirty-eight obese patients diagnosed with PCOS and 35 obese patients without a diagnosis of PCOS were included in the study. Similarly, in our study, the mean MHR value was found to be significantly higher in the PCOS-obese group compared to all other groups $(p=0.004)$. However, in our study, no difference was found between the other groups. This finding might be resulted from the exclusion of patients with metabolic syndrome in our study. For this reason, all these data show that MHR level increases significantly in both those with PCOS and obesity independently from metabolic syndrome. When we excluded the metabolic syndrome, it seemed that only obesity does not have a direct effect on MHR. In addition, the fact that MHR value in non-obese PCOS is not different from non-PCOS groups suggests that obesity triggered by hormonal imbalance in PCOS causes a high increase in MHR value with PCOS.

In our study, the rate of those with a MHR level $\geq 10.1$ was found to be significantly higher in the PCOS-obese group (84.8\% vs $42.5 \%)$ ( $p$ < 0.001). In addition, in logistic regression analysis, it was found that there was a determinability of 7,576 folds (odds ratio; 2.652-21.646) in predicting the presence of PCOS + obesity in those with a MHR value $\geq 10.1$. These findings show that there is a significant relationship between the association of PCOS with obesity and MHR.

When the association of MHR with atherosclerosis and cardiovascular diseases [8] and the relationship between PCOS and obesity and dyslipidemia [31, 32] are evaluated, the fact that MHR has a significant association with both PCOS patients and PCOS-obese patients supports the view that the risk of atherosclerosis and cardiovascular is high with PCOS

In our study, it was found that MHR value was negatively correlated with age in PCOS patients $(p=0.001 ; r=-0.412)$. According to this finding, as age increases, MHR value decreases significantly in patients with PCOS. In our study, the similar age distribution among the groups shows that the relationship between PCOS and MHR is not affected by the age factor. According to these findings, while interpreting the MHR value in patients with PCOS, it should be taken into consideration that the older age may decrease MHR, and cause an incorrect interpretation of the results.

There were some limitations in our study. Since our study was a cross-sectional study, the patients' previous MHR values could not be learned, and the long-term changes in MHR could not be observed, because the patients were not prospectively monitored. In addition, since the long-term risks of cardiovascular diseases could not be observed, this subject could not be evaluated. 


\section{CONCLUSIONS}

MHR is a practical, easy-to-apply, cost-effective and easily calculated indicator that requires no additional testing for patients. Findings obtained from this study showed that MHR level was significantly associated with PCOS, and especially MHR values above 10.1 could be a significant predictive marker of PCOS. Our study findings, in which the metabolic syndrome has been completely excluded, also showed that the association between PCOS and obesity is a very important trigger affecting MHR.

\section{Conflict of interest}

The authors declare that they have no conflict of interest.

\section{REFERENCES}

1. Meier R. Polycystic Ovary Syndrome. Nursing Clinics of North America. 2018; 53(3): 407-420, doi: 10.1016/j.cnur.2018.04.008.

2. Nandi A, Chen Z, Patel R, et al. Polycystic ovary syndrome. Endocrinol Metab Clin North Am. 2014; 43(1): 123-147, doi: 10.1016/j.ecl.2013.10.003, indexed in Pubmed: 24582095.

3. Jakubzick CV, Randolph GJ, Henson PM. Monocyte differentiation and antigen-presenting functions. Nat Rev Immunol. 2017; 17(6): 349-362, doi: 10.1038/nri.2017.28, indexed in Pubmed: 28436425.

4. Ertek S. High-density Lipoprotein (HDL) Dysfunction and the Future of HDL. Curr Vasc Pharmacol. 2018; 16(5): 490-498, doi: 10.2174/1570161 115666171116164612 , indexed in Pubmed: 29149817.

5. Ganjali S, Gotto AM, Ruscica M, et al. Monocyte-to-HDL-cholesterol ratio as a prognostic marker in cardiovascular diseases. J Cell Physiol. 2018; 233(12): 9237-9246, doi: 10.1002/jcp.27028, indexed in Pubmed: 30076716.

6. Zhang Y, LiS, Guo YL, et al. Is monocyte to HDL ratio superior to monocyte count in predicting the cardiovascular outcomes: evidence from a large cohort of Chinese patients undergoing coronary angiography. Ann Med. 2016; 48(5): 305-312, doi: 10.3109/07853890.2016.1168935, indexed in Pubmed: 27087382.

7. Dincgez Cakmak B, Dundar B, Ketenci Gencer F, et al. TWEAK and monocyte to HDL ratio as a predictor of metabolic syndrome in patients with polycystic ovary syndrome. Gynecol Endocrinol. 2019; 35(1): 66-71, doi: 10.1080/09513590.2018.1490401, indexed in Pubmed: 30241442.

8. Usta A, Avci E, Bulbul CB, et al. The monocyte counts to HDL cholesterol ratio in obese and lean patients with polycystic ovary syndrome. Reprod Biol Endocrinol. 2018; 16(1): 34, doi: 10.1186/s12958-018-0351-0, indexed in Pubmed: 29631598.

9. Vahit $D$, Akboga MK, Samet $Y$, et al. Assessment of monocyte to high density lipoprotein cholesterol ratio and lymphocyte-to-monocyte ratio in patients with metabolic syndrome. Biomark Med. 2017; 11(7): 535-540, doi: 10.2217/bmm-2016-0380, indexed in Pubmed: 28685581.

10. Fauser BC, Tarlatzis BC, Rebar RW, et al. Consensus on women's health aspects of polycystic ovary syndrome (PCOS): the Amsterdam ESHRE/ASRM-Sponsored 3rd PCOS Consensus Workshop Group. Fertil Steril. 2012; 97(1): 28-38.e25, doi: 10.1016/j.fertnstert.2011.09.024, indexed in Pubmed: 22153789.

11. FERRIMAN D, GALLWEY JD. Clinical assessment of body hair growth in women. J Clin Endocrinol Metab. 1961; 21: 1440-1447, doi: 10.1210/jcem-21-11-1440, indexed in Pubmed: 13892577.

12. Li Li, Feng Q, Ye M, et al. Metabolic effect of obesity on polycystic ovary syndrome in adolescents: a meta-analysis. J Obstet Gynaecol. 2017; 37(8): 1036-1047, doi: 10.1080/01443615.2017.1318840, indexed in Pubmed: 28657375.

13. Lim SS, Kakoly NS, Tan JWJ, et al. Metabolic syndrome in polycystic ovary syndrome: a systematic review, meta-analysis and meta-regression. Obes Rev. 2019; 20(2): 339-352, doi: 10.1111/obr.12762, indexed in Pubmed: 30339316.

14. Glueck CJ, Goldenberg N. Characteristics of obesity in polycystic ovary syndrome: Etiology, treatment, and genetics. Metabolism. 2019; 92: 108120, doi: 10.1016/j.metabol.2018.11.002, indexed in Pubmed: 30445140.

15. Ammirati E, Moroni F, Magnoni M, et al. Circulating CD14+ and CD14CD16classical monocytes are reduced in patients with signs of plaque neovas- cularization in the carotid artery. Atherosclerosis. 2016; 255 : 171-178, doi: 10.1016/j.atherosclerosis.2016.10.004, indexed in Pubmed: 27751505.

16. Kundi $\mathrm{H}$, Kiziltunc $\mathrm{E}$, Cetin $\mathrm{M}$, et al. Association of monocyte/HDL-C ratio with SYNTAX scores in patients with stable coronary artery disease. Herz. 2016; 41(6): 523-529, doi: 10.1007/s00059-015-4393-1, indexed in Pubmed: 26753671.

17. Murphy AJ, Woollard KJ, Hoang A, et al. High-density lipoprotein reduces the human monocyte inflammatory response. Arterioscler Thromb Vasc Biol. 2008; 28(11): 2071-2077, doi: 10.1161/ATVBAHA.108.168690, indexed in Pubmed: 18617650.

18. Cetin MS, Ozcan Cetin EH, Kalender E, et al. Monocyte to HDL Cholesterol Ratio Predicts Coronary Artery Disease Severity and Future Major Cardiovascular Adverse Events in Acute Coronary Syndrome. Heart Lung Circ. 2016; 25(11): 1077-1086, doi: 10.1016/j.hlc.2016.02.023, indexed in Pubmed: 27118231

19. Wei XB, Chen F, Huang JL, et al. Novel Risk Biomarker for Infective Endocarditis Patients With Normal Left Ventricular Ejection Fraction区- Monocyte to High-Density Lipoprotein Cholesterol Ratio. Circ J. 2017; 82(1): 283-288, doi: 10.1253/circj.CJ-17-0427, indexed in Pubmed: 28781332.

20. Marenzi G, Cosentino N, Bartorelli A. Acute kidney injury in patients with acute coronary syndromes. Heart. 2015; 101(22): 1778-1785, doi: 10.1136/heartjnl-2015-307773.

21. Wekker V, van Dammen L, Koning A, et al. Long-term cardiometabolic disease risk in women with PCOS: a systematic review and meta-analysis. Hum Reprod Update. 2020; 26(6): 942-960, doi: 10.1093/humupd/dmaa029, indexed in Pubmed: 32995872.

22. Amiri $M$, Tehrani FR, Bidhendi-Yarandi $R$, et al. Relationships Between Biochemical Markers of Hyperandrogenism and Metabolic Parameters in Women with Polycystic Ovary Syndrome: A Systematic Review and Meta-Analysis. Horm Metab Res. 2019; 51(1): 22-34, doi: 10.1055/a-08068281, indexed in Pubmed: 30650457.

23. Horne BD, Anderson JL, John JM, et al. Intermountain Heart Collaborative Study Group. Which white blood cell subtypes predict increased cardiovascular risk? J Am Coll Cardiol. 2005; 45(10): 1638-1643, doi: 10.1016/j.jacc.2005.02.054, indexed in Pubmed: 15893180.

24. Ommen SR, Gibbons RJ, Hodge DO, et al. Usefulness of the lymphocyte concentration as a prognostic marker in coronary artery disease. Am J Cardiol. 1997; 79(6): 812-814, doi: 10.1016/s0002-9149(96)00878-8, indexed in Pubmed: 9070569.

25. Khan IM, Pokharel Y, Dadu RT, et al. Postprandial Monocyte Activation in Individuals With Metabolic Syndrome. J Clin Endocrinol Metab. 2016; 101(11): 4195-4204, doi: 10.1210/jc.2016-2732, indexed in Pubmed: 27575945.

26. Johnsen $\mathrm{SH}$, Fosse $\mathrm{E}$, Joakimsen $\mathrm{O}$, et al. Monocyte count is a predictor of novel plaque formation: a 7-year follow-up study of 2610 persons without carotid plaque at baseline the Tromsø Study. Stroke. 2005; 36(4): 715-719, doi: 10.1161/01.STR.0000158909.07634.83, indexed in Pubmed: 15746459 .

27. Akboga MK, Canpolat U, Sahinarslan A, et al. Association of serum total bilirubin level with severity of coronary atherosclerosis is linked to systemic inflammation. Atherosclerosis. 2015; 240(1): 110-114, doi: 10.1016/j.atherosclerosis.2015.02.051, indexed in Pubmed: 25770689.

28. Yayla Ç, Akboğa MK, Gayretli Yayla K, et al. A novel marker of inflammation in patients with slow coronary flow: lymphocyte-to-monocyte ratio. Biomark Med. 2016; 10(5): 485-493, doi: 10.2217/bmm-2016-0022, indexed in Pubmed: 27089433.

29. Ertem AG, Yayla C, Acar B, et al. Relation between lymphocyte to monocyte ratio and short-term mortality in patients with acute pulmonary embolism. Clin Respir J. 2018; 12(2): 580-586, doi: 10.1111/crj.12565, indexed in Pubmed: 27727508.

30. Yue S, Zhang J, Wu J, et al. Use of the Monocyte-to-Lymphocyte Ratio to Predict Diabetic Retinopathy. Int J Environ Res Public Health. 2015; 12(8): 10009-10019, doi: 10.3390/ijerph120810009, indexed in Pubmed: 26308022.

31. Gary T, Pichler M, Belaj K, et al. Lymphocyte-to-monocyte ratio: a novel marker for critical limb ischemia in PAOD patients. Int J Clin Pract. 2014; 68(12): 1483-1487, doi: 10.1111/ijcp.12495, indexed in Pubmed: 25359092.

32. Tedesco S, Adorni MP, Ronda N, et al. Activation profiles of monocyte-macrophages and HDL function in healthy women in relation to menstrual cycle and in polycystic ovary syndrome patients. Endocrine. 2019; 66(2): 360-369, doi: 10.1007/s12020-019-01911-2, indexed in Pubmed: 30993600 